\title{
SNOLA: creando una Red sobre Analíticas de Aprendizaje en España SNOLA: creating a network about Learning Analytics in Spain
}

\author{
Ángel F. Agudo-Peregrina ${ }^{1}$, Manuel Caeiro-Rodríguez ${ }^{2}$, Miguel Á. Conde ${ }^{3}$, Juan Cruz-Benito ${ }^{4}$, Carlos Delgado \\ Kloos $^{5}$, Iratxe Menchaca ${ }^{6}$, Mikel Larrañaga ${ }^{7}$, Alejandra Martínez-Monés ${ }^{8}$, Antonio Robles Gómez ${ }^{9}$ \\ aagudo@tige.ior.etsit.upm.es, manuel.caeiro@det.uvigo.es, mcong@unileon.es, juancb@usal.es, cdk@it.uc3m.es, \\ iratxe.mentxaka@deusto.es, mikel.larranaga@ehu.eus, amartine@infor.uva.es, arobles@scc.uned.es
}

\author{
${ }^{1}$ Departamento de Ingeniería de \\ Organización, Administración de \\ Empresas y Estadística. \\ Universidad Politécnica de Madrid, \\ España
${ }^{4}$ Grupo de Investigación GRIAL.
Departamento de Informática y Automática. \\ Universidad de Salamanca, España
}

\author{
${ }^{2}$ Departamento de Ingeniería \\ Telemática. \\ Universidad de Vigo, España
}

\section{${ }^{5}$ Universidad Carlos III de Madrid,} España

\author{
${ }^{8}$ Departamento de Informática. \\ Universidad de Valladolid, España
}

\begin{abstract}
${ }^{3}$ Dpto. Ingenierías Mecánica, Informática y Aeroespacial. Universidad de León, España
\end{abstract}

\author{
${ }^{6}$ Universidad de Deusto, \\ España
}
${ }^{9}$ Universidad Nacional de Educación a Distancia, España

\begin{abstract}
Resumen- Este artículo introduce de forma somera el Learning Analytics como disciplina y campo de investigación, incluyendo sus principales características, potenciales beneficios de cara a la sociedad, retos y tendencias actuales. A su vez, este manuscrito presenta la Red de Investigación SNOLA (Spanish Network of Learning Analytics, Red Española de Analítica de Aprendizaje), reconocida por el Ministerio de Economía y Competitividad del Gobierno de España. Sobre esta red, se comentan sus objetivos, retos, áreas de trabajo y actividades. En cuanto a SNOLA, se destaca su carácter participativo y abierto hacia la colaboración con distintos actores dentro del área LA, como son las instituciones, usuarios, educadores o los tecnólogos.
\end{abstract}

Palabras clave: analítica de aprendizaje, red de investigación, SNOLA, learning analytics

Abstract- This article briefly introduces Learning Analytics as a discipline and field of research, including its main features, potential benefits for the Society, as well as the current challenges and trends. On the other hand, this manuscript presents the SNOLA Research Network (Spanish Network of Learning Analytics), recognized by the Ministry of Economy and Competitiveness of the Spanish Government. Regarding this research network, the paper discusses its goals, challenges, working areas and activities. Also, is featured the SNOLA participative and open culture towards collaboration with different actors within the LA area, such as institutions, users, educators or technologists.

Keywords: research network, SNOLA, learning analytics

\section{INTRODUCCIÓN}

La sociedad de la información, en la que vivimos en la actualidad, produce una cantidad ingente de datos. Tanto es así, que los datos son parte fundamental en diversos campos como la economía, la gobernanza, el periodismo o la educación (entre muchos otros). La ciencia encargada del tratamiento de esos datos toma varias denominaciones en función del enfoque (i.e. "Big Data”, "Data mining”, "Data Science" etc.) y se aplica de forma habitual en diversos sectores (finanzas, marketing, comunicación, etc.). En el caso de la educación, la conjunción de diversas perspectivas dentro de esa ciencia está resultando en la popularización del término analítica del aprendizaje, (en adelante LA- learning analytics) (Siemens, 2010). En el ámbito de la educación, la disponibilidad de datos e información estructurada en sistemas informáticos ha venido de la mano de la incorporación de las TIC a los procesos de enseñanza-aprendizaje, y más concretamente de los entornos virtuales del aprendizaje (LMS), de plataformas MOOC (siglas en inglés de Cursos Masivos Online Abiertos) u otras, que producen una gran cantidad de datos a través de las interacciones entre usuarios (principalmente estudiantes y profesores), $\mathrm{o}$ de las interacciones entre usuarios $\mathrm{y}$ sistemas $\mathrm{o}$ programas informáticos. Al igual que en otras áreas, existen grandes expectativas sobre los beneficios que el tratamiento de estos información puede aportar a la educación (Ferguson et al., 2016).

Por su naturaleza, el LA debe ser un campo multidisciplinar (Guenaga \& Garaizar, 2015), en el que tecnólogos, pedagogos 
y otros agentes deben colaborar para capturar, procesar, interpretar y aprovechar la información generada desde el punto de vista educativo. Esta perspectiva puramente educativa es lo que diferencia LA de otras disciplinas relacionadas con en el análisis (masivo) de datos. La necesidad de establecer colaboraciones entre distintos actores procedentes de varias disciplinas ha dado lugar a nivel internacional a la aparición de redes de investigación sobre esta temática, LA. Entre ellas se encuentra SNOLA, (Spanish Network of Learning Analytics, Red Española de Analítica del Aprendizaje) ${ }^{1}$ (Acquila-Natale et al., 2017; Caeiro-Rodríguez et al., 2016), que articula una parte importante del trabajo relacionado con LA en nuestro país.

Este artículo presenta las principales características y potenciales beneficios de la analítica del aprendizaje en el aprendizaje y la sociedad, y describe la actividad realizada por la red temática de investigación SNOLA. El objetivo es tender puentes entre dicha red y profesionales de la enseñanza interesadas en aplicar los principios de la LA a la mejora de su actividad docente. La estructura de este artículo es la siguiente: en primer lugar, se describirá de forma breve el campo de la analítica del aprendizaje (learning analytics) y los principales retos a los que se enfrenta. A continuación, se presenta la red temática SNOLA, haciendo hincapié en las posibilidades de colaboración que pueden establecerse entre la red y personas interesadas en colaborar con ella.

\section{CONTEXTO}

Aunque existen diversas definiciones del concepto de analítica del aprendizaje, se puede definir, en línea con lo establecido en el primer congreso de Learning Analytics and Knowledge (LAK) en 2011 (1st Learning Analytics and Knowledge conference, 2011), como la ciencia encargada de la recopilación de datos generados por herramientas educativas (Cruz-Benito, García-Peñalvo, \& Therón, 2014), su transformación, análisis, visualización (Gómez Aguilar, García-Peñalvo, \& Therón, 2014; D. A. Gómez-Aguilar, Conde-González, Theron, \& Garcia-Penalvo, 2011; Diego Alonso Gómez-Aguilar, Hernández-García, García-Peñalvo, \& Therón, 2015; Tobarra, Ros, et al., 2014) e interpretación para poder sacar conclusiones interesantes sobre el proceso del aprendizaje, apoyar en la toma de decisiones y poder mejorarlo (Miguel Ángel Conde \& Hernández-García, 2015; Greller \& Drachsler, 2012).

El campo de la LA está adquiriendo una amplia relevancia en el contexto actual de la tecnología y educación, siendo uno de los términos más en boga en muchas bases de datos y publicaciones científicas (de ámbito generalista o puramente relacionado con la tecnología y la educación). El uso creciente de entornos on-line y TIC para apoyar la enseñanza (sea esta puramente a distancia o presencial), ha dado lugar a una gran disponibilidad de datos acerca de los estudiantes y de sus interacciones con los sistemas (Gašević, Dawson, \& Siemens, 2015). El auge del LA se basa en la idea de que el análisis de estos datos permitirá mejorar el aprendizaje en entornos online y en cualquier proceso de enseñanza-aprendizaje que haga uso de la tecnología (Muñoz-Merino \& Miguez, 2015). Es decir, el análisis de datos permitirá realimentar el proceso de enseñanza, por ejemplo: pudiendo detectar fallos o deficiencias que corregir en la forma en la que los alumnos

${ }^{1}$ SNOLA, http://snola.es aprenden o colaboran para aprender, ayudando en el proceso de aprendizaje de forma activa y en tiempo real, etc.

Un reflejo de esta relevancia que se destaca es el hecho de que el campo de la LA y otros relacionados, como los sistemas adaptativos para el aprendizaje están identificadas como de las más prometedoras en la prestigiosa serie de informes Horizon publicados por el New Media Consortium (Johnson, Adams, \& Cummins, 2013), así como con las estrategias marcadas por la Unión Europea a través de la iniciativa “Opening Up Education” (European Commission, 2016) y el programa Horizon 2020 (European Commission, 2015).

Por otro lado, es creciente también el interés de las empresas por mejorar sus procesos de formación utilizando técnicas de análisis de datos y LA. Por ejemplo, en el evento anual para empresas Next Generation Learning, la temática de LA ha sido introducida en el año 2015 en su $8^{a}$ Edición. Otro ejemplo relevante es el interés mostrado por empresas como IBM, Entelgy, grupo Accenture, Eticas Consulting, etc. en colaborar con entidades que trabajan esta temática, como es esta red SNOLA a través de la organización de actividades de formación o la presentación de productos y estrategias que implementan dichas empresas en los diversos eventos y congresos que se celebran en torno a esta área.

Sin embargo, para cumplir con las expectativas generadas, la comunidad de la LA se enfrenta a varios retos. Por un lado, desafíos tecnológicos y técnicos propios del tratamiento de datos, como su procedencia y naturaleza heterogénea (múltiples fuentes y formatos) (Tobarra, Muñoz, et al., 2014), su calidad, la diversidad de herramientas y técnicas para su procesamiento, y una visualización efectiva para que el usuario final pueda beneficiarse de la información generada. A estos retos tecnológicos se suman los de carácter pedagógico, como la importancia de implicar a docentes y estudiantes en el proceso de LA, la necesidad de generar evidencias que muestren su impacto en la educación, su uso en contextos formales e informales, la identificación de patrones de comportamiento entre los alumnos, o la interpretación pedagógica de la información generada tras el análisis de datos. En relación con estos retos, se puede plantear también el reto de que las instituciones públicas de enseñanza y de gobierno aboguen por implantar este tipo de sistemas a nivel global, de modo que se puedan aprovechar para determinar aspectos como la eficacia del aprendizaje, benchmarks de resultados, etc. Por último, el trabajo en el área ha mostrado lo crucial de enfrentar las cuestiones éticas relacionadas con la privacidad de los datos y con las consecuencias sobre los usuarios del análisis de los datos (Drachsler \& Greller, 2016)

Estos retos se reflejan habitualmente en las áreas temáticas de los congresos, workshops o números especiales de revista específicos de LA (Miguel Á Conde \& Hernández-García, 2013; Delgado Kloos, Essa, \& Muñoz-Merino, 2016; Hernández-García \& Conde, 2014; Muñoz-Merino \& Miguez, 2015; Tobarra, Muñoz, et al., 2014) y en otras más generales de tecnología educativa.

\section{DESCRIPCIÓN}

Los desafíos anteriormente comentados tienen en común la necesidad de incrementar la relación de los grupos de investigación que venían trabajando en analítica del aprendizaje entre sí, y de éstos con los profesores, instituciones, y empresas, con el fin de identificar las 
necesidades de los usuarios y de aprovechar el conocimiento generado para proponer nuevas o mejores formas de hacerles frente.

La necesidad de creación de redes en torno a LA se ha visto a nivel mundial, con proyectos como LACE (proyecto europeo) o de las asociaciones EDM (Educational Data Mining) o SOLAR (Society for Learning Analytics Research). Así mismo, países como Reino Unido y Holanda, ya están creando sus propias redes.

Ante esta demanda a nivel mundial, SNOLA -Spanish Network of Learning Analytics- nace de manera natural e informal en el año 2013, uniendo a investigadores españoles para intercambiar conocimiento y experiencias en el área, así como para establecer sinergias y posibilidades de colaboración. Los principales objetivos de la Red son:

- $\quad$ El fomento de la colaboración entre investigadores de la red, con otros agentes interesados (otros investigadores, empresas e instituciones educativas públicas y privadas) y con otras redes de colaboración europeas e internacionales.

- La difusión y organización de iniciativas relativas a analítica del aprendizaje a escala nacional.

- La puesta a disposición del público de recursos necesarios para la integración eficaz de sistemas de analítica del aprendizaje en los procesos educativos

- La formación de personal investigador y profesionales capacitados para dar respuesta a los nuevos retos de la Sociedad Digital surgidos a partir de la incorporación de la TIC en la educación.

En diciembre de 2015 la red fue reconocida por el Ministerio de Economía y Competitividad como una Red de Excelencia Temática (TIN2015-71669-REDT), incluyendo a nueve universidades españolas: Universidad de Deusto, Universidad de Salamanca, Universidad de León, Universidad del País Vasco (UPV/EHU), Universidad Politécnica de Madrid, Universidad de Valladolid, Universidad Carlos III de Madrid, Universidad de Vigo y Universidad Nacional a Distancia (UNED). Además de estas universidades pertenecientes a la Red de Excelencia reconocida, SNOLA promueve la integración de diversas universidades, empresas y miembros a título individual como colaboradores externos, tal y como se puede observar en la web https://snola.es/miembros/. Como red temática, SNOLA desarrolla una serie de actividades dirigidas a afianzar la comunicación y colaboración entre los miembros de la Red, así como iniciativas para compartir el conocimiento previo con cualquier persona interesada y construir sobre él las soluciones a los retos que actualmente plantea el área de LA.

En la actualidad hay multitud de proyectos aceptados o en marcha que incluyen LA, tanto financiados por la Unión Europea (p.e., LACE, SHEILA, Make World o Go-Lab), por el Ministerio de Economía y Competitividad (p.e., EEE o RESET) o por Comunidades autónomas (p.e. eMadrid). La mayoría de estos proyectos son participados por al menos un grupo de los representados en SNOLA. En algunos de estos proyectos el eje central es el propio LA, mientras que en otros se incorpora como instrumento de mejora de proyectos de tecnología educativa.
En dichos proyectos los integrantes de la red están haciendo frente a los retos presentados por la analítica del aprendizaje desde diferentes perspectivas. Algunos grupos se centran en el uso de LA para el apoyo a métodos de evaluación no tradicionales, como la evaluación de la competencia de trabajo en equipo; algunos grupos han traído el conocimiento adquirido en otros campos relacionados con la LA, como el de los sistemas tutores inteligentes (ITS); o el aprendizaje colaborativo apoyado por ordenador (CSCL). En este último ámbito, se ha investigado como apoyar a profesores de enseñanza universitaria aprovechando sus decisiones de diseño de aprendizaje para hacer más usables los resultados de las herramientas de LA. El uso de LA en contextos reales ha permitido reflexionar sobre los retos a los que se enfrenta el área en su aplicación a niveles educativos diferentes del universitario (Rodríguez-Triana, Martínez-Monés, \& VillagráSobrino, 2016).

\section{Resultados}

La Red se ha marcado como objetivo la atracción de otros agentes (universidades, empresas, administraciones públicas, institutos, asociaciones, etc.) y para dar impulso se han puesto en marcha acciones dirigidas a:

1. Fomento de la movilidad/actividad investigadora.

2. Establecimiento de conexiones, intercambio de conocimiento y colaboraciones a nivel nacional e internacional.

3. Incorporación de nuevos miembros para impulsar sus actividades de $\mathrm{I}+\mathrm{D}+\mathrm{i}$.

Actualmente, SNOLA tiene una página web (http://www.snola.es) donde se aglutinan recursos, herramientas e información para cualquier persona o entidad interesada en el área. En ella se pueden consultar artículos relevantes en el área, proyectos de $\mathrm{I}+\mathrm{D}+\mathrm{i}$, recursos educativos, herramientas software para implementar LA, y las últimas noticias. En ella también se encuentran disponibles los mecanismos para adherirse a la red. Dado el carácter multidisciplinar del área, SNOLA está especialmente interesada en buscar sinergias con actores y grupos de diferentes perfiles, procedentes de los campos de la tecnología, pedagogía, sociología o psicología. La red está interesada en incluir no solo a investigadores, sino también a docentes e instituciones interesados en aplicar los principios de la LA a sus contextos. Por ello cualquier persona, institución o empresa interesada en participar en la Red puede hacerlo, sin coste o restricción alguna. De este modo, la Red cuenta con más de 30 investigadores adheridos a título individual y otras 13 universidades y empresas (además de las 9 que conforman el núcleo de la red reconocida por el Ministerio español y sus investigadores).

SNOLA tiene como uno de sus objetivos prioritarios ser el punto de contacto para la industria, y facilitar el contacto con expertos de la red, de forma que se puedan establecer alianzas y colaboraciones conjuntas para una efectiva transferencia de conocimiento al tejido empresarial. Experiencias anteriores como los LASI 2015 (https://blogs.deusto.es/lasi2015Bilbao/), 2016 (http://lasi16.snola.es/) celebrados en la Universidad de Deusto de Bilbao y el de 2017 (https://lasi17.snola.es/) celebrado en la Universidad Carlos III de Madrid u otras conferencias en las que la red participa (TEEM, SIIE, LAIKA, 
WAPLA, etc.), han demostrado que el número de colaboradores externos en algunos casos podrá superar al de miembros de la Red, lo que demuestra el interés en los temas tratados. Como muestra, se pueden observar ratios de asistencia de más de 50 personas en cada uno de los distintos LASI, o los cientos de asistentes registrados en las otras conferencias en las que participa SNOLA.

Otro de los objetivos de SNOLA es la difusión de conocimiento relacionado con el LA, a través de contenidos en abierto (https://snola.es/software/) y webinars disponibles en su página web, en los cuales referentes nacionales e internacionales aportan su visión y experiencia a cualquier persona interesada en la LA.

\section{CONCLUSIONES}

Este artículo presenta una visión inicial sobre el campo de investigación de la analítica de aprendizaje (learning analytics, LA), comentando sus retos, sus inicios y las principales áreas en las que tiene aplicación o un potencial prometedor. Por otro lado, presenta la red temática española sobre analítica de aprendizaje, SNOLA. Sobre esta red, se comentan sus objetivos, retos, áreas de trabajo y actividades. En cuanto a SNOLA, se destaca su carácter participativo y abierto hacia la colaboración con distintos actores dentro del área LA, como son las instituciones, usuarios, educadores, tecnólogos, etc.

\section{AgRADECIMIENTOS}

La Red de Investigación SNOLA está reconocida oficialmente como una Red Temática de Excelencia (TIN2015-71669-REDT) por el Ministerio de Economía y Competitividad del Gobierno de España, como parte de las acciones de dinamización "Redes de Excelencia" del Programa Estatal de Investigación Científica y Técnica de Excelencia, Subprograma Estatal de Generación de Conocimiento, en el marco del Plan Estatal de Investigación Científica y Técnica y de Innovación 2013-2016.

\section{REFERENCIAS}

1st Learning Analytics and Knowledge conference. (2011). Retrieved from https://tekri.athabascau.ca/analytics/call-papers

Acquila-Natale, E., Caeiro-Rodríguez, M., Conde, M. Á., Cruz-Benito, J., Delgado Kloos, C., Guenaga, M., . . . Robles Gómez, A. (2017). Learning Analytics, o cómo sacar valor a los datos en la educación. Paper presented at the VI Congreso Internacional Multidisciplinar de Investigación Educativa (CIMIE’17), Bilbao, España.

Caeiro-Rodríguez, M., Conde, M. Á., Guenaga, M., Hernández-García, Á., Larrañaga, M., MartínezMonés, A., . . . Rodríguez-Conde, M.-J. (2016). SNOLA: Spanish network of learning analytics. Paper presented at the Proceedings of the Fourth International Conference on Technological Ecosystems for Enhancing Multiculturality.

Conde, M. Á., \& Hernández-García, Á. (2013). A promised land for educational decision-making?: present and future of learning analytics. Paper presented at the Proceedings of the First International Conference on
Technological Ecosystem for Enhancing Multiculturality.

Conde, M. Á., \& Hernández-García, Á. (2015). Learning analytics for educational decision making. Computers in Human Behavior(47), 1-3.

Cruz-Benito, J., García-Peñalvo, F. J., \& Therón, R. (2014). Defining generic data collectors for Learning Analytics: Facing up the heterogeneous data from heterogeneous environments. Paper presented at the International Conference on Advanced Learning Technologies (ICALT) 2014, Athens, Greece. .

Delgado Kloos, C., Essa, A., \& Muñoz-Merino, P. J. (2016). Workshop on Applied and Practical Learning Analytics, WAPLA. Retrieved from http://educate.gast.it.uc3m.es/wapla/

Drachsler, H., \& Greller, W. (2016). Privacy and analytics: it's a DELICATE issue a checklist for trusted learning analytics. Paper presented at the Proceedings of the Sixth International Conference on Learning Analytics \& Knowledge.

European Commission. (2015). Horizon 2020. Retrieved from https://ec.europa.eu/programmes/horizon2020/

European Commission. (2016). Opening up education through new technologies. Retrieved from https://ec.europa.eu/education/policy/strategicframework/education-technology_en

Ferguson, R., Brasher, A., Clow, D., Cooper, A., Hillaire, G., Mittelmeier, J., . . . Vuorikari, R. (2016). Research Evidence on the Use of Learning Analytics: Implications for Education Policy.

Gašević, D., Dawson, S., \& Siemens, G. (2015). Let's not forget: Learning analytics are about learning. TechTrends, 59(1), 64-71.

Gómez Aguilar, D. A., García-Peñalvo, F. J., \& Therón, R. (2014). Analítica Visual en eLearning. El Profesional de la Información, 23(3), 233-242.

Gómez-Aguilar, D. A., Conde-González, M., Theron, R., \& Garcia-Penalvo, F. J. (2011). Reveling the evolution of semantic content through visual analysis. Paper presented at the Advanced Learning Technologies (ICALT), 2011 11th IEEE International Conference on.

Gómez-Aguilar, D. A., Hernández-García, Á., GarcíaPeñalvo, F. J., \& Therón, R. (2015). Tap into visual analysis of customization of grouping of activities in eLearning. Computers in Human Behavior, 47, 6067. doi:http://dx.doi.org/10.1016/j.chb.2014.11.001

Greller, W., \& Drachsler, H. (2012). Translating learning into numbers: A generic framework for learning analytics. Educational Technology \& Society, 15(3), 42-57.

Guenaga, M. L., \& Garaizar, P. (2015). Special Issue. VAEP RITA(http://rita.det.uvigo.es/VAEPRITA/index.php?c ontent $=$ ProxNum\&idiom $=\mathrm{Es})$.

Hernández-García, Á., \& Conde, M. Á. (2014). Dealing with complexity: educational data and tools for learning analytics. Paper presented at the Proceedings of the Second International Conference on Technological Ecosystems for Enhancing Multiculturality.

Johnson, L., Adams, S., \& Cummins, M. (2013). NMC Horizon Report: 2012 Higher Education Edition (Austin, TX: New Media Consortium, 2012).

Octubre 4-6, 2017, Zaragoza, ESPAÑA 
Muñoz-Merino, P. J., \& Miguez, R. (2015). Learning Analytics: impacto, alcance, buenas prácticas y tecnologías. Retrieved from http://www.americalearningmedia.com/edicion038/428-tendencias/6429-learning-analytics-impactoalcance-buenas-practicas-y-tecnologias

Rodríguez-Triana, M. J., Martínez-Monés, A., \& VillagráSobrino, S. (2016). Learning analytics in small-scale teacher-led innovations: ethical and data privacy issues. Journal of Learning Analytics, 3(1), 43-65.

Siemens, G. (2010). What are Learning Analytics? Retrieved from

http://www.elearnspace.org/blog/2010/08/25/whatare-learning-analytics/
Tobarra, L., Muñoz, S. R., Berlinches, R. H., Gómez, A. R., Caminero, A. C., \& Vargas, R. P. (2014). Integration of multiple data sources for predicting the engagement of students in practical activities. IJIMAI, 2(7), 53-62.

Tobarra, L., Ros, S., Hernández, R., Robles-Gómez, A., Caminero, A. C., \& Pastor, R. (2014). Integrated Analytic dashboard for virtual evaluation laboratories and collaborative forums. Paper presented at the Tecnologias Aplicadas a la Ensenanza de la Electronica (Technologies Applied to Electronics Teaching)(TAEE), 2014 XI. 